\title{
Infarto agudo al miocardio en un hombre joven sin ateromatosis coronaria, como forma de presentación de síndrome antifosfolípido primario. Caso clínico
}

\author{
Marisol Jurado $0^{1 a}$, Josefina D urán ${ }^{1 a}$, Alejandro \\ Martínez ${ }^{2}$, José Miguel Castellón ${ }^{2}$, Miguel A Gutiérrez ${ }^{1}$. \\ Acute myocardial infarction in a man \\ without coronary atheromatosis \\ and antiphospholipid syndrome. \\ Report of one case
}

Coronary thrombosis as a manifestation of the antiphospholipid syndrome is very uncommon. We report a 25 year-old male without known cardiovascular risk factors that suffered an acute myocardial infarction as the initial manifestation of the antiphospholipid syndrome. His coronary angiogram demonstrated a single thrombotic lesion in the anterior descending artery without coronary atheromatosis. Anticardiolipin, anti $ß 2$ Glycoprotein I antibodies, and lupus anticoagulant were all positive. Besides the usual management of the coronary thrombosis, the patient was treated with permanent oral anticoagulation. Three months later, a CT coronary angiogram showed complete reperfusion of the involved artery (Rev Méd Chile 2009; 137: 1478-81).

(Key words: Anticoagulants; Antiphospholipid syndrome; Myocardial infarction)

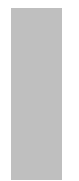
Recibido el 17 de marzo, 2009. Aceptado el 16 de septiembre, 2009.
${ }^{1}$ Departamentos de Inmunología Clínica y Reumatología, Pontificia Universidad Católica de Chile. ${ }^{2}$ Departamento de Enfermedades Cardiovasculares, Pontificia Universidad Católi- ca de Chile. Santiago de Chile.
aResidente de Inmunología Clínica y Reumatología

تlimp síndrome antifosfolípido (SAF) es una causa importante de hipercoagulabilidad adquirida. Se caracteriza por trombosis vascular tanto venosa como arterial y pérdida fetal recurrente en el caso de las mujeres. Para su diagnóstico se utilizan

Correspondencia a: Marisol Jurado O. E mail: marisoljurado@gmail.com. Miguel Gutiérrez. Marcoleta 350, Santiago. Fax: 6324937. E mail: gutierrez@med.puc.cl. tanto criterios clínicos como serológicos. Dentro de los criterios serológicos se requiere la presencia de anticoagulante lúpico (LAC), anticardiolipinas (ACL) IgG o IgM (títulos mayores a 40 GPL o MPL) o anti ß2-glicoproteína-I (ß2-GPI) IgG o IgM positivos (+) (títulos mayores a percentil 99) en al menos 2 oportunidades, separadas por 12 semanas según los criterios de Sapporo modificados ${ }^{1,2}$. La presencia de al menos un criterio clínico y uno de laboratorio es necesaria para el diagnóstico de 
SAF. Ocurre en $1 \%$ y $6 \%$ de la población general y en $50 \%$ de los casos está asociado a lupus eritematoso sistémico (LES) ${ }^{3}$. Las localizaciones de trombosis más frecuentes son el territorio venoso de extremidades inferiores y en segundo lugar el territorio arterial cerebral ${ }^{4}$. La trombosis en el territorio coronario es poco frecuente, aunque la presencia de anticuerpos antifosfolípidos parece ser una causa importante de infarto agudo al miocardio (IAM) en pacientes menores de 40 años ${ }^{5}$.

Presentamos el caso clínico de un paciente de 25 años que presentó un IAM por trombosis arterial coronaria en ausencia de ateromatosis, como manifestación inicial de SAF.

\section{CASO CĹNICO}

Hombre de 25 años con historia de eritromelalgia mano izquierda en la infancia. Sin factores de riesgo cardiovascular conocidos ni consumo de drogas, que en octubre de 2006 tuvo dolor transitorio retroesternal opresivo asociado al esfuerzo. El electrocardiograma no mostró isquemia. Dos meses después presentó dolor de similares características, de $3 \mathrm{~h}$ de duración y consultó a las $24 \mathrm{~h}$ de evolución. El electrocardiograma mostró supradesnivel ST de V2 a V5, onda T negativa de V2 a V6 y aVL y onda Q en V2 - V3. Las enzimas cardíacas estaban elevadas con troponina hasta 35,54 ng/ml. El ecocardiograma evidenció hipoki- nesia marcada no adelgazada anteroseptal distal, anterior y apical. El examen físico era normal, incluyendo las cifras de presión arterial. El perfil lipídico fue normal así como también el hemograma, incluyendo recuento plaquetario. La coronariografía realizada a las 48 h mostró una lesión trombótica de $80 \%$ del tercio proximal de la arteria descendente anterior, sin encontrarse lesiones ateromatosas (Figuras 1A, 1B). Por un TTPK basal prolongado se estudió la presencia de trombofilia y se encontró un anticoagulante lúpico repetidamente positivo (tiempo veneno de serpiente Russell), anticuerpos anticardiolipinas (ACL) IgG 20,6 GPL, IgM 96 MPL (ELISA Louisville APL Diagnostics, Inc, Seabrook, TX, USA) (valor normal (VN) IgG: 0 - 15 GPL; IgM: 0 - $15 \mathrm{MPL}$ ), anticuerpos anti ß2-glicoproteína-I IgG 18,6 U/ml, IgM 77 U/ml (ELSA Binding site, Birmingham, UK) (VN: IgG - IgM: 0 - $20 \mathrm{U} / \mathrm{ml}$ ) y anticuerpos antinucleares $1 / 1280$ patrón mixto. El antiADN y perfil ENA fueron negativos y los complementos normales. Se diagnosticó un SAF primario y se indicó anticoagulación inicialmente con heparina y posteriormente con anticoagulante oral que se mantuvo en forma permanente.

A los 3 meses se realizó angio TAC coronario que mostró reperfusión completa del vaso comprometido (Figura 2). Dos años después se encontraba asintomático, en tratamiento con atenolol, ramipril, aspirina e hidroxicloroquina (Plaquinol ${ }^{\circledR}$ ) además de anticoagulación oral permanente. El ecocardiograma mostró una hipokinesia discreta

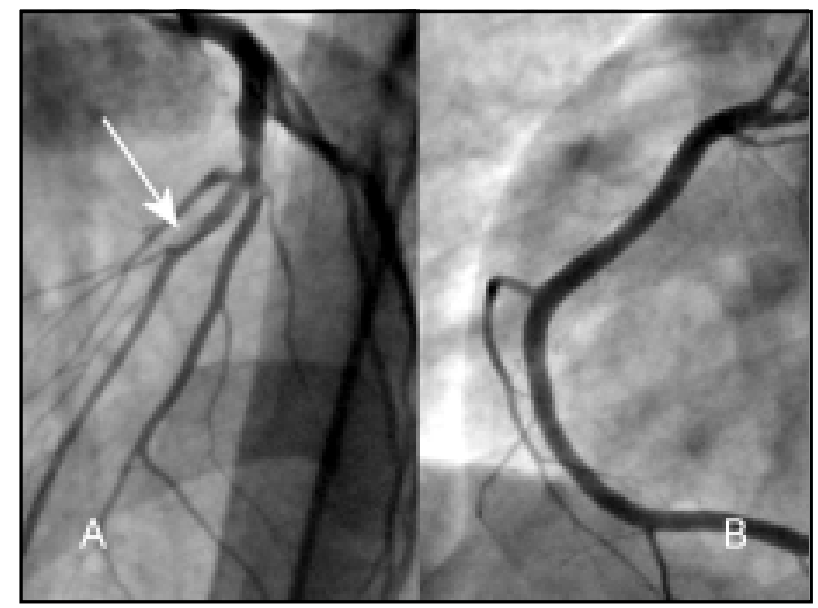

Figura 1. Coronariografía realizada a las $48 \mathrm{~h}$ de evolución del infarto. A: Trombosis de $80 \%$ del lumen de arteria descendente anterior (flecha). B: Arteria coronaria derecha sin lesiones ateromatosas. 


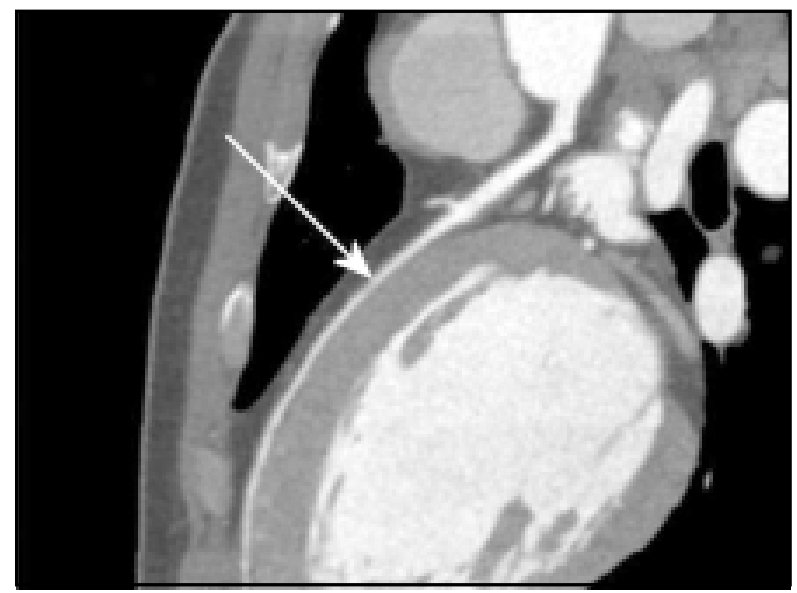

Figura 2. Angio TAC coronario a los 3 meses postinfarto que muestra reperfusión completa de la arteria descendente anterior (flecha).

anteroapical y función sistólica normal baja con fracción de eyección de 53\% por Simpson.

\section{Discusión}

El IAM en los menores de 40 años representa alrededor de 3\% de los casos de enfermedad coronaria $^{6}$. En este grupo de pacientes, es más frecuente encontrar otras causas distintas a la enfermedad ateromatosa coronaria. En los casos con coronariografía normal y ausencia de abuso de drogas como la cocaína, el SAF parece ser una de las más importantes 7,8 .

En este paciente el IAM fue la primera manifestación del SAF y excepto por la presencia de un trombo, las coronarias eran estructuralmente normales sin evidencia de ateroesclerosis.

El IAM es una manifestación poco frecuente del SAF y excepcionalmente puede ser su manifestación inicial. En una cohorte de 1.000 pacientes europeos con SAF la frecuencia de IAM como manifestación inicial fue de 2,8\%, mientras que se presentó durante la evolución del síndrome en 5,5\% de los pacientes ${ }^{4}$, cifra que puede llegar a 20\%-23\%, dependiendo de la severidad de la enfermedad de base, tipo y título de autoanticuerpo ${ }^{3,5}$.

La prevalencia de anticuerpos anticardiolipina (ACL) en pacientes con IAM varía entre 5\% y 15\%, frecuencia que pone en duda al menos en parte su patogenicidad en la población general. En LES su prevalencia es más elevada, por lo que su asociación con la patogenia de la trombosis coronaria es más fuerte ${ }^{9}$. El isotipo IgG en título mayor a 33-40 GPL estaría significativamente asociado al riesgo de trombosis al igual que la presencia de LAC $(+)^{10}$. A pesar de que el isotipo IgG es característicamente más patogénico, está bien descrita la asociación de manifestaciones de SAF en pacientes con isotipo IgM en ausencia de IgG, $12,2 \%$ en una serie de 1.000 pacientes $^{4}$. La presencia de VDRL falso positivo es otra expresión de laboratorio de los aPL, observada en un porcentaje variable de los pacientes ${ }^{11}$. En nuestro paciente no se realizó esta determinación.

La presencia de anti ß2 glicoproteína-I no se ha asociado a desarrollo de IAM en estudios clínicos, sin embargo, estudios in vitro sugieren que al igual que los ACL estaría involucrado en aterogénesis prematura y trombogénesis ${ }^{9,12,13}$. Sin embargo, el riesgo aumentado de IAM en pacientes con SAF está dado más que nada por trombosis coronaria y no por ateroesclerosis prematura ${ }^{14,15}$.

Se pueden plantear tres formas en las que los anticuerpos antifosfolípidos se pueden relacionar con la enfermedad coronaria. La primera por producción de trombosis en arterias coronarias normales, como es el caso de nuestro paciente ${ }^{7}$; la segunda por la eventual asociación de los anticuerpos con ateroesclerosis acelerada ${ }^{16}$; y la última por la inducción transitoria de anticuerpos secundario a la necrosis celular que ocurre en el $\mathrm{IAM}^{17}$. La persistencia de los anticuerpos antifosfolípidos en el tiempo en nuestro paciente, hace improbable que sean secundarios al daño celular del IAM.

En el SAF se han propuesto distintos mecanismos de trombosis; inhibición de precalicreína, interferencia con la activación de los anticoagulantes naturales proteína $\mathrm{C}$ y proteína $\mathrm{S}$, activación endotelial por parte de los anticuerpos antifosfolípidos con inducción de la expresión de moléculas de adhesión y aumento de adhesión de monocitos a las células endoteliales y aumento de la agregación plaquetaria ${ }^{14,18-20}$.

El SAF se considera como primario cuando no está asociado con una enfermedad autoinmune 0 secundario cuando el paciente tiene una enfermedad autoinmune subyacente, usualmente LES. Este 
paciente, a pesar de tener títulos altos de anticuerpos antinucleares, no tenía anticuerpos antiADN ni ninguna manifestación clínica de LES u otra enfermedad del tejido conectivo, por lo que se consideró SAF primario ${ }^{3}$. Si bien es cierto, la progresión de SAF primario a LES es inusual, está descrito que hasta 8\% de los pacientes pueden desarrollarlo durante la evolución de su enfermedad ${ }^{21}$. Nuestro paciente no ha desarmollado manifestaciones clínicas ni alteraciones de laboratorio que hagan sospechar un LES, en un periodo de seguimiento de 3 años.

\section{REFERENCIAS}

1. Wilson WA, Gharavi AE, Koike MD, Lockshi T, Branch DW, PIETTE JC ET AL. International consensus statement on preliminary classification criteria for definite antiphospholipid syndrome: report of an international workshop. Arthritis Rheum 1999; 42: 1309-11.

2. Miyakis S, Lockshin T, Atsumi DW, Branch RL, Brey R, CERVERA ET AL. International consensus statement on an update of the classification criteria for definite antiphospholipid syndrome (APS). J Thromb Haemost 2006; 4: 295-306.

3. Levine JS, Branch DW, Rauch J. The antiphospholipid syndrome. N Engl J Med 2002; 346: 752-63.

4. Cervera R, Piette JC, Font J, Khamashta MA, Shoenfeld Y, CAMPS MT ET AL. Antiphospholipid syndrome: clinical and immunologic manifestations and patterns of disease expression in a cohort of 1,000 patients. Arthritis Rheum 2002; 46: 1019-27.

5. Hamsten A, Norberg R, Bjorkholm M, De Faire U, HoLm G. Antibodies to cardiolipin in young survivors of myocardial infarction: an association with recurrent cardiovascular events. Lancet 1986; 1: 113-6.

6. JALOWIEC DA, Hш JA. Myocardial infarction in the young and in women. Cardiovasc Clin 1989; 20: 197-206.

7. Davies JO, Hunt BJ. Myocardial infarction in young patients without coronary atherosclerosis: assume primary antiphospholipid syndrome until proved otherwise. Int J Clin Pract 2007; 61: 379-84.

8. De Vries PA, Van Der Sluis A, Van Der Horst JC, Van VeLDHUISEN DJ. Myocardial ischaemia with a normal coronary angiogram due to the primary antiphospholipid syndrome. Int J Cardiol 2002; 82: 183-4.

9. VAarala O. Antiphospholipid antibodies and myocardial infarction. Lupus 1998; 7 Suppl 2: S132-4.

10. GaLi M, Luciani D, Bertolini G, Barbui T. Lupus anticoagulants are stronger risk factors for thrombosis than anticardiolipin antibodies in the antiphospholipid syndrome: a systematic review of the literature. Blood 2003; 101: 1827-32.

11. AL Атtia HM. False positive VDRL (BFP-STS) and systemic lupus erythematosus; new data in clinico-
Existe un amplio espectro de otras manifestaciones cardíacas asociadas al SAF como las lesiones valvulares, trombos intracavitarios, miocardiopatía e hipertensión pulmonar ${ }^{14,22}$.

En pacientes jóvenes con IAM, sin factores de riesgo cardiovascular, debe considerarse al SAF entre los diagnósticos diferenciales. Las manifestaciones clínicas y el tratamiento inicial del IAM en pacientes con SAF no varían con respecto a la población general, salvo por la indicación de tratamiento anticoagulante oral a permanencia.

laboratory associations. Int J Dermatol 2002; 41: 858-62.

12. Vaarala O, Puurunen M, Manttari M, Manninen V, Aho $\mathrm{K}$, Palosuo T. Antibodies to prothrombin imply a risk of myocardial infarction in middle-aged men. Thromb Haemost 1996; 75: 456-9.

13. Hasunuma Y, Matsuura E, Makita Z, Katahira T, Nishi S, KoIKE T. Involvement of beta 2-glycoprotein I and anticardiolipin antibodies in oxidatively modified low-density lipoprotein uptake by macrophages. Clin Exp Immunol 1997; 107: 569-73.

14. Abu Romeh IS, Chhatriwala AK, Atik FA, Bhatt DL, NAviA JL. Myocardial infarction secondary to a coronary ostial thrombus in antiphospholipid syndrome. Ann Thorac Surg 2007; 83: 1170-1.

15. Petri M. The lupus anticoagulant is a risk factor for myocardial infarction (but not atherosclerosis): Hopkins Lupus Cohort. Thromb Res 2004; 114: 593-5.

16. VaArala O. Antiphospholipid antibodies and atherosclerosis. Lupus 1996; 5: 442-7.

17. Sietnes KE, Smith P, Abdelnoor M, Arnesen H, Wisloff F. Antiphospholipid antibodies after myocardial infarction and their relation to mortality, reinfarction, and non-haemorrhagic stroke. Lancet 1992; 339: 451-3.

18. Ogimoto A, Sekiya M, Funada J, Miyagawa M, Kubo Y, AKUTSU H. Antiphospholipid syndrome with acute myocardial infarction and portal vein occlusion: a case report. Jpn Circ J 2000; 64: 468-70.

19. Asherson RA, Khamashta MA, Baguley E, Oakiey CM, ROWELL NR, Hughes GR. Myocardial infarction and antiphospholipid antibodies in SLE and related disorders. Q J Med 1989; 73: 1103-15.

20. Roubey RA, HofFman M. From antiphospholipid syndrome to antibody-mediated thrombosis. Lancet 1997; 350: 1491-3.

21. Gómez-Puerta JA, Martin H, Amgo MC, Aguirre MA, CAMPs MT, Cuadrado MJ ET AL. Long-term follow-up in 128 patients with primary antiphospholipid syndrome: do they develop lupus? Medicine (Baltimore) 2005; 84: 225-30.

22. Osula S, BeLl GM, Hornung RS. Acute myocardial infarction in young adults: causes and management. Postgrad Med J 2002; 78: 27-30. 